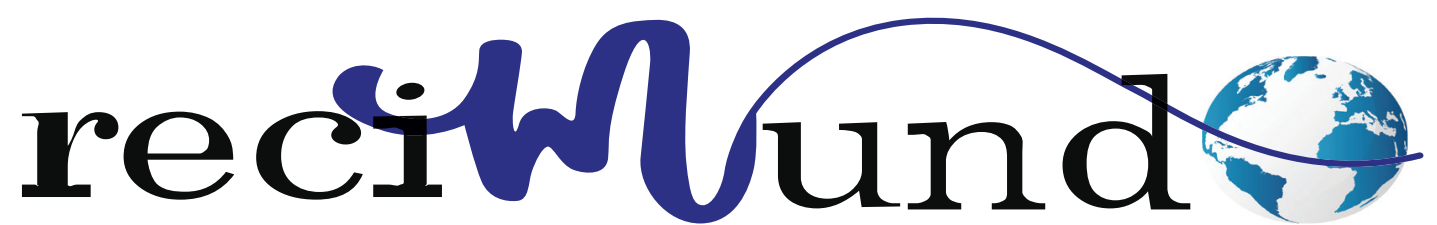

Revista Científica Mundo de la Investigación y el Conocimiento

DOI: 10.26820/recimundo/4.(4).octubre.2020.4-12

URL: http://recimundo.com/index.php/es/article/view/876

EDITORIAL: Saberes del Conocimiento

REVISTA: RECIMUNDO

ISSN: 2588-073X

TIPO DE INVESTIGACIÓN: Artículo de Revisión

CÓDIGO UNESCO: 23 Química

PAGINAS: 4-12

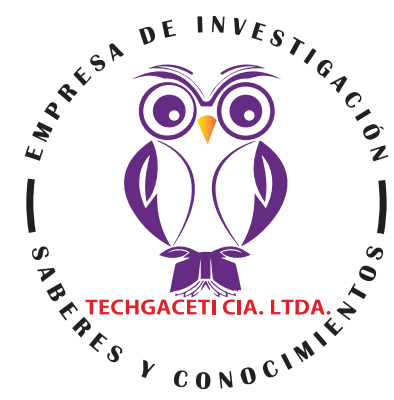

\title{
Plasma acoplado inductivamente en espectroscopia de emisión optica (ICP-OES)
}

Inductively coupled plasma in optical emission spectroscopy (ICP-OES)

Plasma indutivamente acoplado em espectroscopia de emissão óptica (ICP-OES)

María Elena Jiménez Heinert'; Ana de las Mercedes Grijalva Endara²; Henry Xavier Ponce Solórzano³

RECIBIDO: 10/07/2020 ACEPTADO: 26/08/2020 PUBLICADO: 15/10/2020

1. Magister en Química Farmacéutica, Doctora en Química y Farmacia; Químico Farmacéutico; Docente de la Universidad de Guayaquil; Guayaquil, Ecuador; marelejh@hotmail.com; (ID) https://orcid.org/0000-0003-3482-6233

2. Magister en Cambio Climático; Magister en Auditoria de la Gestión de Calidad; Diploma Superior de Auditoria de Gestión de la Calidad; Química Farmacéutica; Docente de la Facultad de Ciencias Químicas; Universidad de Guayaquil; Guayaquil, Ecuador; ana.grijalvae@ug.edu.ec; (D) https://orcid.org/0000-0003-4143-5863

3. Magister en Tecnología e Innovación Educativa, Ingeniero en Electrónica y Telecomunicaciones; Docente de la Universidad de Guayaquil; Guayaquil, Ecuador; henry.ponces@ug.edu.ec; ID https://orcid.org/0000-0003-2029-8601

\section{CORRESPONDENCIA \\ María Elena Jiménez Heinert \\ marelejh@hotmail.com}

Guayaquil, Ecuador 


\section{RESUMEN}

La espectroscopia de emisión óptica con plasma acoplado inductivamente (ICP-OES, por sus siglas en inglés), es una de las técnicas más usadas en todo el mundo para determinar trazas de elementos en una gran variedad de matrices, las cuales comúnmente deben digerirse previamente. Hoy en día, es una técnica sólida y ampliamente usada en muchos laboratorios con la finalidad de realizar análisis rutinarios. Entre las principales áreas que emplean esta técnica se encuentran: agricultura y alimentos, geología, aguas y en el campo de la medicina. El objetivo del presente estudio consiste en plasmar aspectos generales acerca de la espectroscopia de emisión óptica por plasma acoplado inductivamente, tales como la espectroscopia de emisión (OES), espectroscopía de emisión óptica por plasma acoplado inductivamente (ICPOES), la fuente de plasma acoplada inductivamente, principios de funcionamiento, aplicaciones, ventajas y desventajas. El modelo de investigación es una revisión de tipo documental bibliográfico. Entre las principales ventajas se encuentran una menor interferencia entre elementos consecuencia directa de sus temperaturas más elevadas y buenos espectros para la mayoría de los elementos con unas mismas condiciones de excitación, en consecuencia, el registro simultáneo de los espectros para docenas de elementos. Como toda técnica también tiene sus desventajas, entre las que se encuentran ser una técnica de alto costo de adquisición y mantenimiento, requieren pretratamiento de muestra, además de capacitación especializada para su manejo. El camino por recorrer en la investigación que puede aportar la IPC-OES es muy largo, el avance de la tecnología alrededor de esta técnica pronostica un panorama positivo en la mejora del estudio de la materia, no obstante, es fundamental el conocimiento y manejo de los conceptos actuales de esta técnica.

Palabras clave: Espectroscopia, Emisión, Plasma, Acoplado, Inductivamente.

\section{ABSTRACT}

Inductively Coupled Plasma Optical Emission Spectroscopy (ICP-OES) is one of the most widely used techniques around the world to determine trace elements in a wide variety of matrices, which commonly must be previously digested. Today, it is a robust technique widely used in many laboratories for routine testing purposes. Among the main areas that use this technique are: agriculture and food, geology, water and in the field of medicine. The objective of this study is to capture general aspects about inductively coupled plasma optical emission spectroscopy, such as emission spectroscopy (OES), inductively coupled plasma optical emission spectroscopy (ICP-OES), the plasma source inductively coupled, operating principles, applications, advantages and disadvantages. The research model is a bibliographic documentary type review. Among the main advantages are lower interference between elements, a direct consequence of their higher temperatures and good spectra for most elements with the same excitation conditions, consequently, the simultaneous recording of spectra for dozens of elements. Like any technique, it also has its disadvantages, including being a high acquisition and maintenance cost technique, requiring sample pre-treatment, as well as specialized training for handling. The way to go in the research that the IPC-OES can contribute is very long, the advancement of technology around this technique predicts a positive panorama in the improvement of the study of the matter, however, the knowledge and management of current concepts of this technique.

Keywords: Spectroscopy, Emission, Plasma, Coupled, Inductively.

\section{RESUMO}

A espectroscopia de emissão óptica com plasma indutivamente acoplado (ICP-OES) é uma das técnicas mais amplamente utilizadas em todo o mundo para determinar oligoelementos em uma ampla variedade de matrizes, que geralmente devem ser previamente digeridas. Hoje, é uma técnica robusta amplamente utilizada em muitos laboratórios para fins de testes de rotina. Entre as principais áreas que utilizam esta técnica estão: agricultura e alimentação, geologia, água e no campo da medicina. O objetivo deste estudo é capturar aspectos gerais sobre espectroscopia de emissão óptica com plasma indutivamente acoplado, como espectroscopia de emissão (OES), espectroscopia de emissão óptica com plasma indutivamente acoplado (ICP-OES), fonte de plasma indutivamente acoplada, princípios de operação, aplicações, vantagens e desvantagens. O modelo de pesquisa é uma revisão bibliográfica do tipo documental. Entre as principais vantagens estão a menor interferência entre os elementos, consequência direta de suas temperaturas mais elevadas e bons espectros para a maioria dos elementos com as mesmas condições de excitação, conseqüentemente, o registro simultâneo de espectros para dezenas de elementos. Como toda técnica, também tem suas desvantagens, inclusive ser uma técnica de alto custo de aquisição e manutenção, exigindo pré-tratamento da amostra, além de treinamento especializado para manuseio. O caminho a percorrer nas pesquisas que o IPC-OES pode contribuir é muito longo, o avanço da tecnologia em torno desta técnica prevê um panorama positivo no aprimoramento do estudo do assunto, porém, o conhecimento e gestão dos conceitos atuais deste técnica.

Palavras-chave: Espectroscopia, Emissão, Plasma, Acoplada, Indutivamente. 


\section{Introducción}

Las técnicas espectrométricas que se basan en plasma, en la actualidad, son ampliamente usadas en el campo del análisis elemental. La instrumentación utilizada para dichas técnicas, ha evolucionado mucho a lo largo de los años, fundamentalmente por el desarrollo en los componentes electrónicos.

La espectroscopia es un campo bastante amplio, con muchas subdisciplinas, que pueden clasificarse según el tipo de material que se analice. "La espectroscopia atómica incluye un número de técnicas analíticas utilizadas para determinar la composición elemental de una muestra examinando su espectro electromagnético o su espectro de masas". Entre estas técnicas se encuentra la espectroscopia de emisión atómica, que pueden ser: MP-AES, Fluorescencia por rayos $X(X R F)$ y la ICP-OES. (Agilent Technologies, 2016)

Acerca de la espectrometría de emisión óptica con plasma acoplado inductivamente (ICP-OES por sus siglas en inglés), Calderilla Jaimes (2018) refiere que esta técnica espectroscópica se basa en un plasma altamente energético y eléctricamente neutro, el cual se encuentra compuesto por iones, electrones y partículas neutras, comúnmente de argón, y recibe la energía de un campo electromagnético de alta frecuencia o de una corriente continua, que puede llegar a alcanzar una temperatura de hasta 8,000 ${ }^{\circ}$ K. (p. 21)

Con relación a los antecedentes históricos de esta técnica, Aguirre Pastor (2015) destaca:

Esta técnica fue desarrollada por Greenfield et al. en el año 1964, apoyada en los trabajos de Reed, quien usara un plasma de acoplamiento inductivo, por primera vez, cuyos objetivos específicos no contemplaban los espectrométricos. En 1974 se co- mercializó el primer equipo de ICP-OES y, desde entonces, su desarrollo ha crecido exponencialmente. En sus inicios, la técnica tenía una serie de inconvenientes, como los relativamente altos límites de detección (>ug g) para muchas muestras con concentraciones ultra bajas (<ng g-1) en los analitos de interés. Para solventar esta vicisitud, Houk desarrolló en su tesis doctoral el primer ICP-MS, basándose en trabajos anteriores de Gray, el cual había desarrollado un espectrómetro de masas usando un plasma de corriente continua. La técnica fue bien aceptada por la comunidad científica y en 1983 se introdujo en el mercado los primeros ICP-MS patrocinados por Sciex (Perkin Elmer Corporation Ltd.) y por VG (Fisons Instruments Ltd.). (p. 15, 16)

Hoy en día, es una técnica sólida y ampliamente usada en muchos laboratorios en todo el mundo con la finalidad de realizar análisis rutinarios. Entre las áreas que emplean esta técnica se encuentran: agricultura y alimentos, geología, aguas y en el campo de la medicina (Universidad de Alicante, 2020). Esta técnica resulta excelente en el campo de la medicina por su especial precisión y sensibilidad en el rastreo de impurezas presentes en medicamentos y productos biológicos. "Más investigación y nutrición lo convertirán en uno de los instrumentos líderes en los sectores farmacéutico y biomédico y más confiable para garantizar la seguridad de la salud de los pacientes". (Khan, 2019)

El conocimiento de esta técnica es sumamente importante en muchas áreas de nuestra vida cotidiana, son muchos los aportes que las investigaciones con espectroscopia y especialmente con ICP-OES se han realizado y es mucho todavía el camino que recorrer.

El objetivo del presente estudio consiste en plasmar aspectos generales acerca de la espectroscopia de emisión óptica por plasma acoplado inductivamente, tales 
como la espectroscopia de emisión (OES), espectroscopía de emisión óptica por plasma acoplado inductivamente (ICP-OES), la fuente de plasma acoplada inductivamente, principios de funcionamiento, aplicaciones y ventajas y desventajas.

\section{Materiales y métodos}

A los fines de llevar a cabo el presente estudio se llevó a cabo una búsqueda de material bibliográfico digital, que representó la base fundamental para el análisis y compendio del tema de la espectroscopia de emisión óptica con plasma acoplado inductivamente (ICP-OES). En virtud de lo cual, la presente investigación se clasifica como de tipo documental o bibliográfica.

Dicha búsqueda se llevó a cabo por medio de diferentes bases de datos y páginas web que están asociadas con el área de la salud, tanto de carácter nacional como internacional, con reconocida validez científica. Entre las principales bases de datos fueron usadas las siguientes: SciELO, Redalyc, Medigraphic, entre otros. El tipo de material bibliográfico consistió en informes, tesis de grado, tesis de maestrías y otras clases de contenidos. En cuanto a los contenidos repetidos, estos fueron desestimados, así como editoriales o cartas editoriales, anotaciones académicas y cualquier otro tipo de material bibliográfico carente de fuentes de sustento científico o con bajo nivel de evidencia. La búsqueda se llevó a cabo durante la última quincena del mes de septiembre del presente año. Los descriptores usados fueron los siguientes: "Espectroscopia", "Espectroscopia de emisión"; "Espectroscopia de emisión óptica" e "Espectroscopia de emisión óptica de plasma acoplado inductivamente". Los registros bibliográficos se filtraron en base a los criterios de idioma español e inglés, relevancia del tema, con una correlación temática y actuales. Tanto la metodología como el análisis y la argumentación expuestos en el presente trabajo, se llevaron a cabo de manera consensuada entre los miembros del equipo investigador.

\section{Resultados}

\section{Espectroscopia de emisión (OES)}

Para Rinaldi (2015) la espectroscopía de emisión (OES) presenta las siguientes características:

La OES se basa en la producción y detección de espectros de línea emitidos durante el proceso de desexcitación radiactiva de electrones que sufren transiciones entre niveles excitados superiores y niveles excitados inferiores o el nivel fundamental. Esos electrones pertenecen a las órbitas externas de los átomos y se llaman electrones ópticos. Los espectros de línea son específicos de cada elemento y la adecuada selección de una línea y su aislamiento por medio de un sistema dispersivo permite al analista verificar la presencia de ese elemento y determinar su concentración. Un espectrómetro de emisión atómica por plasma inductivo de argón consiste en una fuente de radiación, un sistema de introducción de muestras, un sistema óptico dispersivo, un detector y la correspondiente electrónica para adquisición, procesamiento y edición de resultados (ver figura 1). (p. 37) 


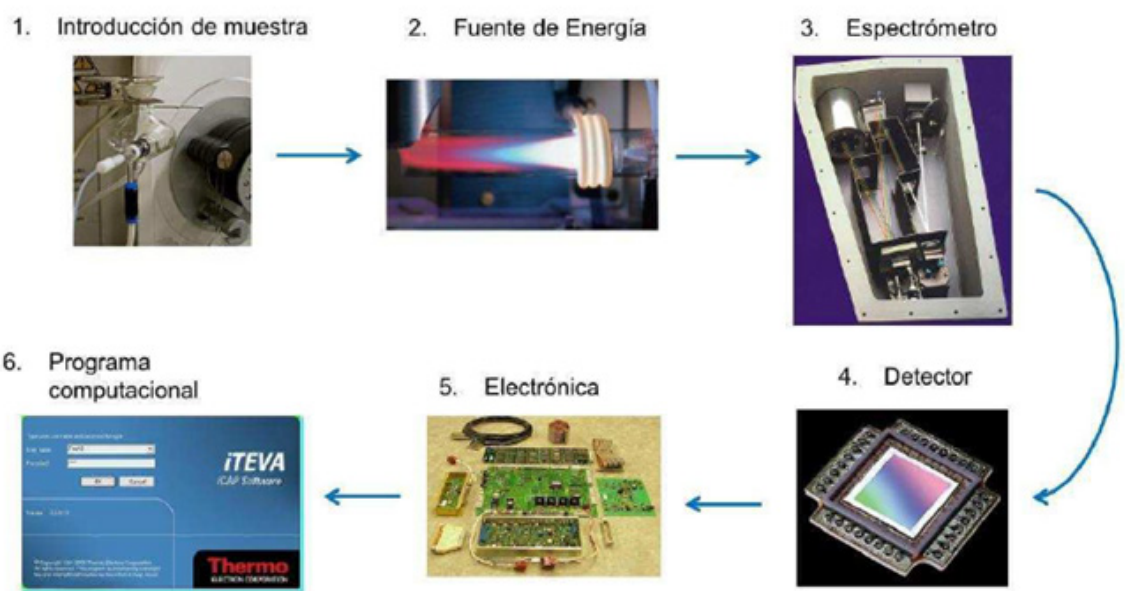

Figura 1. Esquema simplificado de los componentes de un ICP-OES. Recuperado de: "Estudio de los contenidos de elementos inorgánicos de interés ambiental en suelos afectados por derrames de combustibles derivados del petróleo".

Fuente: Rinaldi, D. S. (2015). p. 39. Facultad de Ciencias Exactas y Naturales. Universidad de Buenos Aires. Recuperado de: https://bibliotecadigital.exactas.uba.ar/download/ tesis/tesis_n5829_Rinaldi.pdf

\section{Espectroscopía de emisión óptica por plasma acoplado inductivamente (ICP- OES)}

La espectroscopia de emisión óptica con plasma acoplado inductivamente (ICP-OES, por sus siglas en inglés), según Jimeno, 2017 citado por Zamora, Lozano, Samayoa, Velásquez, \& Prado (2019) refiere que es una de las técnicas más usadas para determinar trazas de elementos en una gran variedad de matrices, las cuales comúnmente deben digerirse previamente. asimismo, agrega:

La ICP-OES se basa en la emisión espontánea de fotones de los átomos que han sido excitados por un plasma de argón de alta temperatura (6800 K), cuya señal es característica de cada elemento y en ciertas condiᄀciones proporcional a la concentración. Los equipos analíticos actuales permiten la medición simultánea de más de 70 elementos, aunque los parámetros de desempeño de la técnica deben evaluarse para cada una de las matrices de trabajo, ya que existen inter $\neg$ ferencias espectrales que deben corregirse. (p. 47)

\section{La fuente de plasma acoplada inductiva- mente}

La fuente de plasma también conocida como antorcha, se puede definir de la siguiente manera:

Tres tubos concéntricos de cuarzo por medio de los cuales fluye una corriente de argón, con un caudal total comprendido entre 11 y $17 \mathrm{~L} / \mathrm{min}$.; el diámetro del tubo más grande es aproximadamente de $2,5 \mathrm{~cm}$. Rodeando la parte superior de este tubo se encuentra una bobina de inducción refrigerada por agua, alimentada por un generador de radiofrecuencia capaz de producir una potencia de $2 \mathrm{kw}$ a unos $27 \mathrm{MHz}$. La ionización del argón que fluye se inicia por medio de una chispa que proviene de una bobina Tesla. Los iones resultantes y sus electrones asociados interaccionan entonces en el campo magnético oscilante que se produce por la bobina de inducción. Esta interacción hace que los iones y los electrones dentro de la bobina se muevan 
en trayectorias anulares cerradas; el calentamiento óhmico es una consecuencia de la resistencia a este movimiento. (Rinaldi, 2015, p. 41, 42)

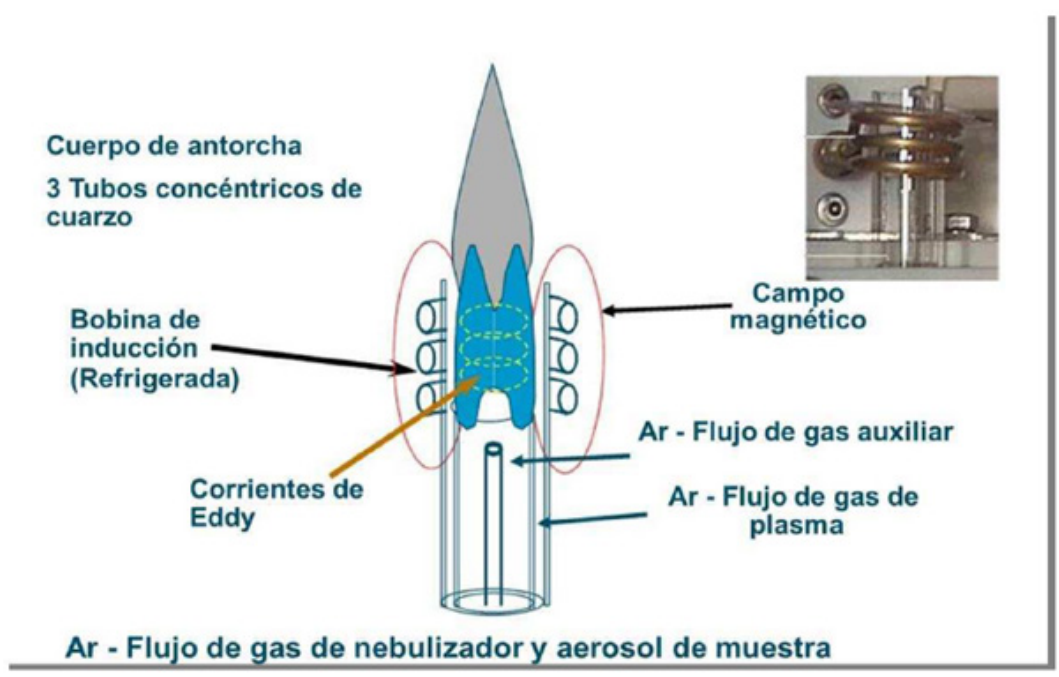

Figura 2. Antorcha típica para una fuente de plasma acoplado inductivamente. Recuperado de: "Estudio de los contenidos de elementos inorgánicos de interés ambiental en suelos afectados por derrames de combustibles derivados del petróleo".

Fuente: Rinaldi, D. S. (2015). p. 39. Facultad de Ciencias Exactas y Naturales. Universidad de Buenos Aires. Recuperado de: https://bibliotecadigital.exactas.uba.ar/download/ tesis/tesis_n5829_Rinaldi.pdf

\section{Principios de funcionamiento de la IPC- OES}

Para Fanego Hernández \& Carabeo Pérez (2017), mediante la espectrometría de emisión atómica de plasma acoplado inductivamente "es posible determinar de forma cuantitativa la mayoría de los elementos de la tabla periódica a niveles de traza y ultra-traza, partiendo de muestras en disolución acuosa". Explican dichos autores el proceso de la siguiente manera:

La muestra, en forma líquida, es transportada por medio de una bomba peristáltica hasta el sistema nebulizador donde es transformada en aerosol gracias a la acción de gas argón. Dicho aerosol es conducido a la zona de ionización que consiste en un plasma generado al someter un flujo de gas argón a la acción de un campo magnético oscilante inducido por una corriente de alta frecuencia. En el interior del plasma se pueden llegar a alcanzar temperaturas de hasta 8000 K. En estas condiciones, los átomos presentes en la muestra son ionizados/excitados. Al volver a su estado fundamental, estos iones o átomos excitados emiten radiaciones de una longitud de onda que es característica de cada elemento. Esta radiación pasa a través de un sistema óptico que separa la radiación según su longitud onda. A continuación, un detector mide la intensidad de cada una de las radiaciones relacionando esta con la concentración de cada elemento en la muestra. (p. 55)

\section{Aplicaciones}

En líneas generales, las aplicaciones básicas de la IPC-OES son las siguientes: 
- Monitorización de agua/aguas residuales/residuos sólidos

- Determinación de trazas de elementos en agua

- Monitorización de mercurio en muestras medioambientales

- Análisis cuantitativo de varios elementos en muestras medioambientales de agua/suelo/sedimento

- Análisis de suelo: análisis del contenido de micronutrientes (agricultura)

- Determinación de metales preciosos y oro. (Agilent Technologies, 2016)

Asimismo, mediante ICP-OES se pueden detectar y determinar cuantitativamente la mayoría de los elementos del Sistema Periódico, pudiéndose analizar una amplia variedad de tipos de muestra. Más específicamente tiene sus usos en las áreas que se mencionan a continuación:

- Agricultura y alimentos

- Determinación de metales y posibles contaminantes en suelos, fertilizantes, materias vegetales, alimentos, etc.

- Análisis clínico

- Determinación de elementos tóxicos en orina, sangre, heces, leche materna, tejidos.

- Geología

- Determinación de la procedencia de sedimentos y rocas a través de su composición.

- Evaluación de la contaminación de suelos.

- Aguas

- Determinación de metales y contaminantes en aguas continentales, potables, vertido, salmueras y aguas de mar. (Universidad de Alicante, 2020)

\section{Ventajas y desventajas}

Una de las ventajas principales del ICP sobre otras fuentes de emisión se encuentra derivada de la habilidad de vaporizar, atomizar, excitar y ionizar eficientemente y reproduciblemente en un amplio rango de elementos presentes en diferentes tipos de muestra. Una de las razones importante de la superioridad del ICP sobre la llama u horno es la alta temperatura del plasma. Mientras la llama y el horno alcanzan temperaturas de $3300 \mathrm{~K}$, la temperatura del gas en el centro del plasma es aprox. 6800 K. Además de mejorar la eficiencia en la excitación e ionización, la alta temperatura también reduce o eliminar muchas de las interferencias químicas encontradas en llamas y hornos. Una característica importante del ICP que no es común en otras fuentes de emisión es que cuando los átomos de la muestra llegan al punto de observación del plasma ya han estado un tiempo de residencia de alrededor de 2 milisegundos a temperaturas que van de 6000 a $8000 \mathrm{~K}$. Estos tiempos y temperaturas son de 2 a 3 veces mayores que los que se alcanzan en las llamas más calientes de combustión (acetileno / óxido nitroso). Por lo tanto, la atomización es más completa y se presentan menos interferencias químicas. (Skoog \& Leary, 1994)

Por otra parte, Gomis Yagües (2008) reitera dentro de las principales ventajas de la espectroscopía de emisión atómica basada en la atomización con plasma, las siguientes:

- Menor interferencia entre elementos consecuencia directa de sus temperaturas más elevadas.

- Se pueden obtener buenos espectros para la mayoría de los elementos con unas mismas condiciones de excitación, en consecuencia, es posible registrar simultáneamente los espectros para docenas de elementos.

- Permiten la determinación de bajas concentraciones de elementos que tiende a formar compuestos refractarios, esto es, compuestos que son muy resistentes a la descomposición térmica, tales como $\mathrm{B}, \mathrm{P}, \mathrm{U}, \mathrm{Zr} \mathrm{Nb}$.

- Permiten la determinación de no metales como $\mathrm{Cl}$, Br, I y S. 
- Son aplicables en unos intervalos de concentración que abarcan varios órdenes de magnitud.

- Menos tiempo de análisis.

- En cuanto a sus desventajas, el mismo autor refiere las siguientes:

- Equipo más caro.

- Mayor coste de operación que la espectroscopia de absorción atómica.

- Menor precisión que la espectroscopia de absorción atómica.

Por último, para Calderilla Jaimes (2018) "las principales desventajas de estas técnicas es que son de alto costo de adquisición y mantenimiento, requieren pretratamiento de muestra y capacitación especializada para su manejo". (p. 18)

\section{Conclusiones}

Al poderse excitar la materia con la finalidad de que esta produzca una emisión de radiación, por pequeña que sea, no obstante, capaz de ser percibida, dicha radiación tendrá la capacidad de revelar los sucesos y el comportamiento con la materia. En tal sentido, la espectroscopia de emisión óptica de plasma acoplado inductivamente (ICP-OES) es una de las técnicas más usadas para determinar trazas de elementos en una gran variedad de matrices.

Igualmente, la IPC-OES es ampliamente usada en muchas áreas tales como agricultura y alimentos, geología, aguas, medicina, farmacéutica, entre otros. Las bondades de sus numerosas ventajas superan técnicas similares, entre las principales se encuentran: una menor interferencia entre elementos consecuencia directa de sus temperaturas más elevadas y buenos espectros para la mayoría de los elementos con unas mismas condiciones de excitación, en consecuencia, el registro simultáneo de los espectros para docenas de elementos.

Como toda técnica también tiene sus desventajas, entre las que se encuentran ser una técnica de alto costo de adquisición y mantenimiento, requieren pretratamiento de muestra, además de capacitación especializada para su manejo.

El camino por recorrer en la investigación que puede aportar la IPC-OES es muy largo, el avance de la tecnología alrededor de esta técnica pronostica un panorama positivo en la mejora del estudio de la materia, no obstante, es fundamental el conocimiento y manejo de los conceptos actuales de esta técnica.

\section{Bibliografía}

Agilent Technologies. (14 de Marzo de 2016). agilent. com. Recuperado el 20 de septiembre de 2020, de https://www.agilent.com/cs/library/slidepresentation/public/5991-6593_Agilent_Atomic\%20 Spectroscopy_Hardware_ES.pdf

Aguirre Pastor, M. A. (2015). Avances en la preparación de muestras líquidas para análisis elemental con técnicas espectrométricas basadas en plasma ICP-OES, ICP-MS y LIBS. Tesis Doctoral, Universidad de Alicante, Facultad de ciencias. Departamento de química analítica, nutrición y bromatología, Alicante. Recuperado el 22 de Septiembre de 2020, de https://rua.ua.es/dspace/bitstream/10045/48125/1/tesis_miguel_angel_aguirre_pastor.pdf

Calderilla Jaimes, C. M. (2018). Avances en la determinación de metales basados en la técnica de análisis en flujo multijeringa e impresión 3D. Tesis Doctoral, Universitat de les Illes Balears, Centro de Investigación en Materiales Avanzados S. C., Palma de Mallorca. Recuperado el 28 de Septiembre de 2020, de https://dspace.uib.es/xmlui/bitstream/ handle/11201/150031/Carlos\%20Mauricio\%20 Calderilla\%20Jaime_TESIS\%20DEF.pdf?sequen$\mathrm{ce}=1$ \&isAllowed $=\mathrm{y}$

Fanego Hernández, S., \& Carabeo Pérez, V. (2017). Empleo de técnicas de alta precisión en la industria azucarera para el análisis de mieles finales. Instituto Cubano de Investigaciones de los Derivados de la Caña de Azúcar (ICIDCA), 51(3), 50-57. Recuperado el 23 de Septiembre de 2020, de http:// www.redalyc. org/articulo.oa?id $=223158039008$

Gomis Yagües, V. (2008). Técnicas Instrumentales en el Análisis Industrial. Tema 7. Espectroscopia de emisión y absorción atómica. Guía, Universidad de Alicante, Departamento de Ingeniería Química, Alicante. Recuperado el 24 de septiem- 
bre de 2020, de https://rua.ua.es/dspace/bitstream/10045/8252/4/T7Abasorc.pdf

Khan, K. F. (2019). Application, principle and operation of ICP-OES in pharmaceutical analysis. The Pharma Innovation Journal, 8(11), 281-282. Recuperado el 29 de Septiembre de 2020, de http:// www.thepharmajournal.com/archives/2019/vol8issue11/PartE/8-11-19-350.pdf

Rinaldi, D. S. (2015). Estudio de los contenidos de elementos inorgánicos de interés ambiental en suelos afectados por derrames de combustibles derivados del petróleo. Tesis de Maestría, Facultad de Ciencias Exactas y Naturales, Universidad de Buenos Aires, Buenos Aires. Recuperado el 15 de Septiembre de 2020, de https://bibliotecadigital.exactas.uba.ar/download/tesis/tesis_n5829_Rinaldi.pdf
Skoog, D. A., \& Leary, J. J. (1994). Análisis instrumental (4ta edición ed.). Madrid, España: McGraw-Hill Interamericana.

Universidad de Alicante. (2020). Universidad de Alicante. Recuperado el 22 de Septiembre de 2020, de https://sstti.ua.es/es/instrumentacion-cientifica/unidad-de-analisis/espectroscopia-de-emision-por-plasma-de-acoplamiento-inductivo.html

Zamora, O., Lozano, R., Samayoa, H., Velásquez, M., \& Prado, B. (2019). Adecuabilidad y comparación de técnicas espectroscópicas para el análisis de muestras de orígen geológico. Revista internacional de contaminación ambiental, 35(1), 65-79. Recuperado el 21 de Septiembre de 2020, de http://www.scielo.org.mx/scielo.php?pi$d=$ S0188-49992019000100065\&script=sci_arttext

\section{CITAR ESTE ARTICULO:}

Jiménez Heinert, M., Grijalva Endara, A., \& Ponce Solórzano, H. (2020). Plasma acoplado inductivamente en espectroscopia de emisión óptica (ICP-OES). RECIMUNDO, 4(4), 4-12. doi:10.26820/recimundo/4.(4).octubre.2020.4-12 